\title{
Comparing Jupiter and Saturn: dimensionless input rates from plasma sources within the magnetosphere
}

\author{
V. M. Vasyliūnas \\ Max-Planck-Institut für Sonnensystemforschung, 37191 Katlenburg-Lindau, Germany
}

Received: 25 March 2008 - Accepted: 19 May 2008 - Published: 11 June 2008

\begin{abstract}
The quantitative significance for a planetary magnetosphere of plasma sources associated with a moon of the planet can be assessed only by expressing the plasma mass input rate in dimensionless form, as the ratio of the actual mass input to some reference value. Traditionally, the solar wind mass flux through an area equal to the cross-section of the magnetosphere has been used. Here I identify another reference value of mass input, independent of the solar wind and constructed from planetary parameters alone, which can be shown to represent a mass input sufficiently large to prevent corotation already at the source location. The source rate from Enceladus at Saturn has been reported to be an order of magnitude smaller (in absolute numbers) than that from Io at Jupiter. Both reference values, however, are also smaller at Saturn than at Jupiter, by factors $\sim 40$ to 60 ; expressed in dimensionless form, the estimated mass input from Enceladus may be larger than that from Io by factors $\sim 4$ to 6 . The magnetosphere of Saturn may thus, despite a lower mass input in $\mathrm{kg} \mathrm{s}^{-1}$, intrinsically be more heavily mass-loaded than the magnetosphere of Jupiter.
\end{abstract}

Keywords. Magnetospheric physics (Magnetosphere interactions with satellites and rings; Planetary magnetospheres) - Space plasma physics (Ionization processes)

\section{Introduction}

A planetary magnetosphere may possess sources of plasma deep within its interior volume, in addition to the well-known sources at the exterior boundary (the solar wind) and at the interior boundary (the ionosphere): ionization of neutral particles emanating from the moons of the planet. For Jupiter in particular, it has long been accepted that the primary source of plasma for the magnetosphere is the moon Io, with a mass

Correspondence to: V. M. Vasyliūnas

(vasyliunas@mps.mpg.de) input of about $10^{3} \mathrm{~kg} \mathrm{~s}^{-1}$ (e.g. Hill et al., 1983; Thomas et al., 2004, and references therein). More recently, the moon Enceladus has been found to be a major source of mass, both plasma and neutral particles, for the magnetosphere of Saturn (Dougherty et al., 2006; Tokar et al., 2006; Waite et al., 2006; Hansen et al., 2006); the plasma mass input rate is not as well determined as it is at Jupiter, but a value $\geq 10^{2} \mathrm{~kg} \mathrm{~s}^{-1}$ has been estimated by Pontius and Hill (2006) from plasma flow perturbations. This is an order of magnitude smaller than the corresponding value at Jupiter, and on the basis of this comparison it is sometimes stated that Saturn has a weaker interior plasma source than Jupiter.

The relative importance of a plasma source cannot, however, be judged on the basis of absolute numbers in $\mathrm{kg} \mathrm{s}^{-1}$ or amu s ${ }^{-1}$; rather, the mass input rate must be expressed in dimensionless terms, dividing it by some suitable, physically relevant scale quantity. The purpose of this note is to compare the interior plasma sources of Jupiter and Saturn on a dimensionless basis, in two ways: relative to the solar wind mass flux, and relative to a (newly defined) critical input rate derived from planetary parameters alone.

\section{Scaling to solar wind mass flux}

A widely used comparison of the interior source rate is with the mass flux of the solar wind through an area approximately equal to the projected cross-section of the magnetosphere:

$S_{s w}=\pi\left(\mathrm{R}_{\mathrm{M}} / \mathrm{R}_{\mathrm{P}}\right)^{2} \rho_{s w} \mathrm{~V}_{s w} \mathrm{R}_{\mathrm{P}}{ }^{2}$

where $R_{M}$ is the distance from the planet to the subsolar magnetopause (conventionally expressed in units of the planet's radius $\mathrm{R}_{\mathrm{P}}$ ), $\rho_{s w}$ is the mass density and $\mathrm{V}_{s w}$ the bulk flow speed of the solar wind. Note: the amount of solar wind plasma entering the magnetosphere is generally assumed to be a small fraction of $S_{s w}$, of order $O\left(10^{-3}\right)$ for Earth (e.g. Hill, 1979b, and references therein). The solar wind density decreases with increasing distance $r$ from the Sun as 
$1 / r^{2}$. At Saturn in comparison to Jupiter, $\rho_{s w}$ is thus smaller by a factor $(5.2 / 9.5)^{2} \simeq 0.30,\left(R_{M} / R_{P}\right)^{2}$ is smaller by a factor approximately $\sim 1 / 9$ and $\left(\mathrm{R}_{\mathrm{P}}\right)^{2}$ is smaller by a factor $(60268 / 71400)^{2} \simeq 0.71$, giving all told

$S_{s w}($ Saturn $) / S_{s w}($ Jupiter $) \sim 0.024 \sim 1 / 42$.

The reference solar wind mass flux is smaller at Saturn than at Jupiter primarily because of the lower solar wind density as well as the smaller size of the magnetosphere.

\section{Scaling to planetary parameters}

At both Jupiter and Saturn, the plasma injection process occurs deep within the magnetosphere, where the influence of the solar wind may be minimal. It is thus natural to ask: can the mass input rates be compared on the basis of planetary parameters only, with no reference to the solar wind? The primary given parameters are the distance $r_{s}$ of the source (orbital distance of the moon), the planetary rotation rate $\Omega\left(=2 \pi / \tau_{\text {rot }}\right)$, and the planet's magnetic moment $\mu$. The latter is usually expressed as $\mu=\mathrm{B}_{\mathrm{P}} \mathrm{R}_{\mathrm{P}}{ }^{3}$, where $\mathrm{B}_{\mathrm{P}}$ is the surface magnetic field strength at the equator; for most purposes in the magnetosphere, however, given a fixed $\mu$, the separate values of $\mathrm{B}_{\mathrm{P}}$ and $\mathrm{R}_{\mathrm{P}}$ do not matter except for a few special aspects (e.g. Vasyliūnas, 2004).

From the parameters $r_{s}, \Omega$, and $\mu$, one can construct one quantity having the dimensions of mass per unit time:

$S_{c r}=\frac{\mu^{2}}{\Omega r_{s}{ }^{5}}$.

The physical meaning of this critical mass input rate is seen more easily if Eq. (2) is rewritten in an expanded form,

$S_{c r}=4 \pi r_{s}^{2}\left[\frac{1}{8 \pi}\left(\frac{\mu}{r_{s}{ }^{3}}\right)^{2} \frac{1}{(1 / 2)\left(\Omega r_{s}\right)^{2}}\right] \Omega r_{s}$

(Gaussian units): $S_{c r}$ is the mass outflow when the mass density equals the energy density of the magnetic field at the equator divided by the corotational kinetic energy per unit mass, and the outflow speed equals the corotation speed, all at the source distance $r_{s}$. In Sect. 4, I show that a mass input $S_{c r}$ implies breakdown of corotation already at the distance $r_{s}$.

Expressed in numerical terms,

$S_{c r}=5.73 \times 10^{9} \mathrm{~kg} \mathrm{~s}^{-1}\left(\frac{\mathrm{B}_{\mathrm{P}}}{1 \text { Gauss }}\right)^{2} \frac{\tau_{\mathrm{rot}}}{10 \mathrm{~h}} \frac{\mathrm{R}_{\mathrm{P}}}{10^{4} \mathrm{~km}}\left(\frac{\mathrm{R}_{\mathrm{P}}}{r_{s}}\right)^{5}$

giving, with $r_{s}=5.91 \mathrm{R}_{\mathrm{J}}$ for Io and $r_{s}=3.94 \mathrm{R}_{\mathrm{S}}$ for Enceladus,

$S_{c r}($ Jupiter $) \simeq 1.03 \times 10^{8} \mathrm{~kg} \mathrm{~s}^{-1}$

$S_{c r}($ Saturn $) \simeq 1.76 \times 10^{6} \mathrm{~kg} \mathrm{~s}^{-1}$,

or

$S_{c r}($ Saturn $) / S_{c r}($ Jupiter $) \sim 0.017 \sim 1 / 59$.
The critical mass flux is smaller at Saturn than at Jupiter primarily because of the much smaller magnetic dipole moment.

\section{Relation of $S_{c r}$ to breakdown of corotation}

The critical distance $R_{0}$, beyond which the dipole field is too weak to exert the centripetal acceleration required for maintaining the plasma in rigid corotation, can be estimated as

$R_{0}{ }^{4} \simeq \mu^{2} /\left(\pi r_{s}^{3} \Omega^{2} h_{s} \rho_{s}\right) \simeq 2 \mu^{2} /\left(r_{s} \Omega^{2} M\right)$

where $\rho_{s}$ is the plasma density and $h_{s}$ the plasma sheet thickness at the source distance $r_{s}$ (see detailed derivation in Vasyliūnas, 1983, and references therein), and the second expression follows by noting that $M \simeq 2 \pi r_{s}^{2} h_{s} \rho_{s}$ is approximately the total mass in the plasma sheet. From Eq. (4),

$$
\left(\frac{R_{0}}{r_{s}}\right)^{4} \simeq \frac{\mu^{2}}{\Omega r_{s}{ }^{5}} \frac{2}{\Omega M}=\frac{2 S_{c r}}{\Omega M},
$$

hence a mass input equal to $S_{c r}$ accumulates, during approximately one rotation period, a mass sufficiently large to break open the magnetic field lines at the source distance $r_{s}$.

Another limit to corotation, independent of Eq. (5), is set by the finite Pedersen conductance $\Sigma_{P}$ of the planet's ionosphere. With a mass input $S$ that must be transported outward, plasma flow becomes markedly subcorotational beyond a distance $R_{H}$ (Hill, 1979a)

$R_{H}{ }^{4} \simeq \pi \Sigma_{P} \mu^{2} / c^{2} S$

which can be rewritten with the use of Eq. (2) as

$$
\begin{aligned}
\left(\frac{R_{H}}{r_{s}}\right)^{4} & \simeq \frac{\pi \Sigma_{P} \Omega r_{s}}{c^{2}} \frac{S_{c r}}{S} \\
& \simeq \frac{\Sigma_{P}}{1 \mathrm{mho}} \frac{\Omega r_{s}}{3184 \mathrm{~km} \mathrm{~s}^{-1}} \frac{S_{c r}}{S}
\end{aligned}
$$

or, inserting the values of $\Omega r_{s}$ at Io and Enceladus, respectively,

$$
\begin{aligned}
\left(\frac{R_{H}}{r_{s}}\right)^{4} & \simeq \frac{\Sigma_{P}}{56 \mathrm{mho}} \frac{S_{c r}}{S} \quad \text { (Jupiter) } \\
& \simeq \frac{\Sigma_{P}}{120 \mathrm{mho}} \frac{S_{c r}}{S} \quad \text { (Saturn) }
\end{aligned}
$$

For any plausible value of $\Sigma_{P}, S=S_{c r}$ implies $R_{H}<r_{s}$ : a mass input $S_{c r}$ is too large to be accelerated to rigid corotation, even at the source location. The assumption implicit in deriving Eq. (5), that rigid corotation is maintained until the magnetic field lines break open, is therefore not valid; what remains true in any case, however, is that a mass input $S_{c r}$ does not allow rigid corotation anywhere. 


\section{Comparison with observations}

There is a long-standing ambiguity in the use of the terms "mass input rate" or "mass loading rate" applied to plasma sources within planetary magnetospheres, which must be taken note of when comparing the observed values in the magnetospheres of Jupiter and Saturn. Strictly speaking (narrow sense), these terms should denote processes that add plasma to the system: photoionization and electron-impact ionization of the neutral particles (Eq. (6) holds only with $S$ meant in this sense). In practice, however, the terms are widely used also (broad sense) to denote all ionization processes, including charge exchange between plasma and neutral particles which does not result in a net addition of plasma but only in a net escape of neutrals. The distinction is particularly significant at Saturn, where the abundance ratio of plasma to neutral particles is much lower than at Jupiter ( Jurac and Richardson, 2005; Delamere et al., 2007).

The dimensionless expressions are applicable, of course, for the mass input rate in either the narrow or the broad sense; one just has to make sure, when comparing Jupiter and Saturn, that the reported observed values are meant in the same sense for both planets. The canonical value of $\sim 10^{3} \mathrm{~kg} \mathrm{~s}^{-1}$ for Jupiter is unquestionably the plasma mass input rate in the broad sense (cf. Thomas et al., 2004, Table 23.1). It can therefore properly be compared with the rate $\sim 10^{2} \mathrm{~kg} \mathrm{~s}^{-1}$ (minimum value) inferred by Pontius and Hill (2006) from plasma momentum loading and thus also representing the plasma mass input rate in the broad sense.

\section{Conclusions}

Although the magnetospheric mass input rate inferred from observations is smaller by (at least) an order of magnitude at Saturn compared to that at Jupiter, this does not necessarily imply (as often supposed) that the magnetosphere of Saturn must be mass-loaded to a lesser extent than that of Jupiter. On the contrary, when expressed in appropriate dimensionless variables, the mass input at Saturn may very well be larger than that at Jupiter, because the scale quantities are also much smaller at Saturn than they are at Jupiter: the solar wind mass flux incident on the projected magnetospheric area $S_{s w}$ is smaller by a factor $\sim 40$, and the critical mass flux $S_{c r}$ that would completely prevent corotation is smaller by a factor $\sim 60$.

As discussed in Sect. 5, the observed mass loading (in the broad sense, including charge exchange) at Saturn may be consistently estimated as smaller by a factor $\sim 10$ than the canonical value of the mass loading at Jupiter. Relative to $S_{s w}$, mass loading at Saturn then exceeds that at Jupiter by a factor $\sim 4$. Relative to $S_{c r}$, mass loading at Saturn exceeds that at Jupiter by a factor $\sim 6$. At both planets, the actual mass input rates are smaller than $S_{c r}$ by many orders of magnitude, but Saturn's is closer by a factor $\sim 6$ to its $S_{c r}$ than Jupiter's is to its. Enceladus may thus be a more significant source of plasma for the magnetosphere of Saturn than Io is for the magnetosphere of Jupiter.

Acknowledgements. I am grateful to A. J. Dessler, T. W. Hill, and the referee for useful comments.

Topical Editor I. A. Daglis thanks one anonymous referee for her/his help in evaluating this paper.

\section{References}

Delamere, P. A., Bagenal, F., Dols, V., and Ray, L. C.: Saturn's neutral torus versus Jupiter's plasma torus, Geophys. Res. Lett., 34, L09105, doi:10.1029/2007GL029437, 2007.

Dougherty, M. K., Khurana, K. K., Neubauer, F. M., Russell, C. T., Saur, J., Leisner, J. S., and Burton, M. E.: Identification of a dynamic atmosphere at Enceladus with the Cassini magnetometer, Science, 311, 1406-1409, doi:10.1126/science.1120985, 2006.

Hansen, C. J., Esposito, J. L., Stewart, I. F., Colwell, J., Hendrix, A., Pryor, W., Shemansky. D., and West, R.: Enceladus' water vapor plume, Science, 311, 1422-1425, doi:10.1126/science.1121254, 2006.

Hill, T. W.: Inertial limit on corotation, J. Geophys. Res., 84, 65546558, 1979a.

Hill, T. W.: Rates of mass, momentum, and energy transfer at the magnetopause, in: Magnetospheric Boundary Layers, edited by Battrick, B. and Mort, J., 325-332, ESA SP-148, 1979 b.

Hill, T. W., Dessler, A. J., and Goertz, C. K.: Magnetospheric models, in: Physics of the Jovian Magnetosphere, edited by: Dessler, A. J., 395-453, Cambridge University Press, N.Y., 1983.

Jurac, S. and Richardson, J. D.: A self-consistent model of plasma and neutrals at Saturn: Neutral cloud morphology, J. Geophys. Res., 110, A09220, doi:10.1029/2004JA010635, 2005.

Pontius Jr., D. H. and Hill, T. W.: Enceladus: A significant plasma source for Saturn's magnetosphere, J. Geophys. Res., 111, A09214, doi:10.1029.2006JA011674, 2006.

Thomas, N., Bagenal, F., Hill, T. W., and Wilson, J. K.: The Io neutral cloud and plasma torus, in: Jupiter, edited by: Bagenal, F., Dowling, T. E., and McKinnon, W. B., 561-591, Cambridge University Press, N.Y., 2004.

Tokar, R. L., Johnson, R. E., Hill, T. W., Pontius, D. H., Kurth, W. S., Crary, F. J., Young, D. T., Thomsen, M. F., Reisenfeld, D. B., Coates, A. J., Lewis, G. R., Sittler, E. C., and Gurnett, D. A.: The interaction of the atmosphere of Enceladus with Saturn's plasma, Science, 311, 1409-1412, doi:10.1126/science.1121061, 2006.

Vasyliūnas, V. M.: Plasma distribution and flow, in: Physics of the Jovian Magnetosphere, edited by: Dessler, A. J., 395-453, Cambridge University Press, N.Y., 1983.

Vasyliūnas, V. M.: Comparative magnetospheres: lessons for Earth, Adv. Space Res., 33, 2113-2120, 2004.

Waite Jr., J. H., Combi, M. R., Ip, W.-H., Cravens, T. E., McNutt Jr., R. L., Kasprzak, W., Yelle, R., Luhman, J., Niemann, H., Gell, D., Magee, B., Fletcher, G., Lunine, J., and Tseng, W.-L.: Cassini Ion and Neutral Mass Spectrometer: Enceladus plume composition and structure, Science, 311, 1419-1422, doi:10.1126/science.1121290, 2006. 